\title{
Clinical Practice Patterns of Radiotherapy in Patients with Hepatocellular Carcinoma: A Korean Radiation Oncology Group Study (KROG 14-07)
}

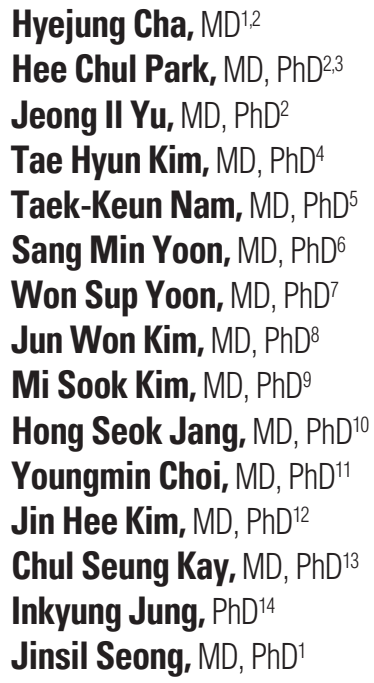

${ }^{*}$ A list author's affiliations appears at the end of the paper.

\begin{abstract}
Purpose
The aim of this study was to examine patterns of radiotherapy (RT) in Korean patients with hepatocellular carcinoma (HCC) according to the evolving guideline for HCC established by the Korean Liver Cancer Study Group-National Cancer Center (KLCSG-NCC).
\end{abstract}

\section{Materials and Methods}

We reviewed 765 patients with HCC who were treated with RT between January 2011 and December 2012 in 12 institutions.

\section{Results}

The median follow-up period was 13.3 months (range, 0.2 to 51.7 months). Compared with previous data between 2004 and 2005, the use of RT as a first treatment has increased (9.0\% vs. 40.8\%). Increased application of intensity-modulated RT resulted in an increase in radiation dose (fractional dose, 1.8 Gy vs. 2.5 Gy; biologically effective dose, 53.1 Gy $_{10}$ vs. $56.3 \mathrm{~Gy}_{10}$ ). Median overall survival was 16.2 months, which is longer than that reported in previous data (12 months). In subgroup analysis, treatments were significantly different according to stage $(p<0.001)$. Stereotactic body RT was used in patients with early HCC, and most patients with advanced stage were treated with three-dimensional conformal RT.

\section{Conclusion}

Based on the evolving KLCSG-NCC practice guideline for HCC, clinical practice patterns of RT have changed. Although RT is still used mainly in advanced HCC, the number of patients with good performance status who were treated with RT as a first treatment has increased. This change in practice patterns could result in improvement in overall survival.

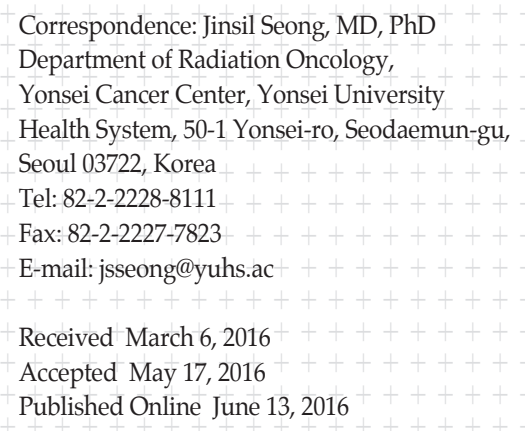

\section{Key words}

Hepatocellular carcinoma, Radiotherapy,

Clinical practice pattern

\section{Introduction}

Liver cancer is one of the leading primary cancers and the main cause of death in Korea. The 5-year survival rates have improved in liver cancer patients diagnosed from 2008 to 2012 compared with those diagnosed from 1993 to 1995. However, the prognosis of liver cancer is still poor compared to that of other cancer patients [1].
The Korean Liver Cancer Study Group (KLCSG) and the National Cancer Center, Korea (NCC) developed a guideline for the management of hepatocellular carcinoma (HCC) in 2003 [2]. This guideline provided recommendations for the diagnosis and treatment of patients with newly diagnosed HCC. The indications of radiotherapy (RT) were as follows: (1) definitive aim: Child-Pugh (C-P) class A or B, tumor less than two-thirds of the whole liver, and no extrahepatic metastasis; (2) palliative aim: pain caused by tumor, tumor 
expected to rupture, portal vein tumor thrombosis, bile duct obstruction due to tumor, or extrahepatic metastasis.

After the development of this guideline, a national profile of HCC patients who received RT between January 2004 and December 2005 was reported [3]. Data from 10 institutions were analyzed and 398 HCC patients were treated with RT. Median age was 57 years and $85 \%$ of patients were male. Three fourths of patients had C-P class A and the Eastern Cooperative Oncology Group (ECOG) performance status was $0-1$ in 234 patients $(58.8 \%)$. Most patients $(78 \%)$ were in an advanced stage (stage III or IV), and 27 and 162 patients had lymph node (LN) metastasis and portal vein thrombosis (PVT), respectively. RT was used mainly as a salvage treatment after the failure of other treatments and was performed predominantly using three-dimensional conformal RT (3D CRT). Median total and fractional dose were $45 \mathrm{~Gy}$ and 1.8 Gy, respectively. Median survival was 12 months and the overall survival (OS) at 2 years was $27.9 \%$. Tumor size, LN metastasis, and biological effective dose (BED) were significant factors of OS, and C-P class A was an additional significant factor in 326 patients treated with 3D CRT. Evaluation of treatment outcome was available in 145 patients (36.4\%).

After that, many studies regarding treatment outcome of RT in Korean patients with HCC have been reported in Korea. In 2009, the Clinical Practice Guideline for HCC was revised by the KLCSG-NCC [4].

The aim of this study was to examine patterns of RT in patients with HCC in Korea according to the evolving guideline for HCC established by the KLCSG-NCC in 2009. The secondary aim was to analyze treatment outcome and to find out predictive clinical factors.

\section{Materials and Methods}

Patients diagnosed with HCC by pathological confirmation or radiological finding with an elevation of tumor marker by KLCSG-NCC guideline and treated with RT for HCC between January 2011 and December 2012 were eligible for this study. RT was performed for primary HCC, PVT, or regional LN metastasis. Exclusion criteria were presence of distant metastasis, double primary cancer within 5 years, or history of irradiation to the liver. Finally, we reviewed 765 patients with HCC who were treated with RT in 12 participating institutions.

Data regarding patient, tumor, treatment, survival, and treatment failure were collected. The modified International Union for Cancer Control (mUICC) staging system that the guideline recommended as a primary staging system was used. Treatment data included RT technique, total radiation
Table 1. Patient and tumor characteristics

\begin{tabular}{|c|c|}
\hline Characteristic & $\begin{array}{l}\text { No. }(\%) \\
(\mathrm{n}=765)\end{array}$ \\
\hline Age, median (range, yr) & $57(24-86)$ \\
\hline \multicolumn{2}{|l|}{ Sex } \\
\hline Male & $641(83.8)$ \\
\hline Female & $124(16.2)$ \\
\hline \multicolumn{2}{|l|}{ Viral type } \\
\hline B & $596(77.9)$ \\
\hline $\mathrm{C}$ & $69(9.0)$ \\
\hline B \& C & $5(0.7)$ \\
\hline NBNC & $86(11.2)$ \\
\hline Other & $9(1.2)$ \\
\hline \multicolumn{2}{|l|}{ ECOG } \\
\hline $0-1$ & $714(93.3)$ \\
\hline $2-3$ & $51(6.7)$ \\
\hline \multicolumn{2}{|l|}{ C-P class } \\
\hline A & $615(80.4)$ \\
\hline B & $128(16.7)$ \\
\hline C & $14(1.8)$ \\
\hline Unknown & $8(1.0)$ \\
\hline \multicolumn{2}{|l|}{ Pre-RT treatment } \\
\hline No & $312(40.8)$ \\
\hline Yes & $453(59.2)$ \\
\hline \multicolumn{2}{|l|}{ mUICC stage } \\
\hline I & $29(3.8)$ \\
\hline II & $111(14.5)$ \\
\hline III & $287(37.5)$ \\
\hline IV & $338(44.2)$ \\
\hline Tumor size $^{\text {a) }}$, median (range, $\mathrm{cm}$ ) & $6.5(0-20.0)$ \\
\hline \multicolumn{2}{|l|}{ No. of tumors } \\
\hline Single & $315(41.3)$ \\
\hline Multiplicity & $448(58.7)$ \\
\hline \multicolumn{2}{|l|}{ PVT } \\
\hline No & $389(50.8)$ \\
\hline Yes & $376(49.2)$ \\
\hline \multicolumn{2}{|l|}{ LN metastasis } \\
\hline No & $667(87.2)$ \\
\hline Yes & $98(12.8)$ \\
\hline \multicolumn{2}{|l|}{ Pre-RT tumor marker } \\
\hline AFP, median (range, IU/mL) & $95.3(0.3-2,922,754.0)$ \\
\hline $\begin{array}{l}\text { PIVKA-II, median } \\
\text { (range, } \mathrm{mAU} / \mathrm{mL} \text { ) }\end{array}$ & $283.5(7.0-176,595.0)$ \\
\hline
\end{tabular}

NBNC, non-B non-C; ECOG, Eastern Cooperative Oncology Group; C-P, Child-Pugh; RT, radiotherapy; mUICC, modified International Union for Cancer Control; PVT, portal vein thrombosis; LN, lymph node; AFP, alphafetoprotein; PIVKA-II, protein induced by vitamin $\mathrm{K}$ absence-II. a Tumor size: intrahepatic tumor size based on modified Response Evaluation Criteria in Solid Tumors Criteria. 
dose, fractional dose, and combination with other treatment modality. Combination treatment was defined as a treatment administered within 4 weeks of the start of RT or the end of RT. Total radiation dose was converted to BED and the $\alpha / \beta$ ratio was constrained to be $10 \mathrm{~Gy}$.

Treatment failure was defined as progression of disease according to the modified Response Evaluation Criteria in Solid Tumors and was categorized into infield and outfield failures. Infield failure was defined as a treatment failure in the RT field, and outfield failure included outfield-intrahepatic and extrahepatic failures.

OS was calculated from the start day of RT to death, and failure-free survival (FFS) was calculated from the start day of RT to treatment failure. Survival was estimated using the Kaplan-Meier method. Predictive factors of survival were analyzed using the Cox proportional hazard model, and multivariate analysis was performed using stepwise regression. Age, tumor size, pre-RT $\alpha$-fetoprotein (AFP), and BED were analyzed as continuous variables. Radiotherapy characteristics according to stage were analyzed using the Kruskal-Wallis test, and statistical analysis was performed using SPSS ver. 20.0 (IBM Corp., Armonk, NY).

\section{Results}

The median follow-up period for all patients was 13.3 months (range, 0.2 to 51.7 months). Patient and tumor characteristics are shown in Table 1 . The median age was 57 years (range, 24 to 86 years), and 641 patients were male $(83.8 \%)$. Most patients (93.3\%) had ECOG score $0-1$, and $80.4 \%$ were C-P class A; $82 \%$ of patients were diagnosed as mUICC stage III or IV. Median tumor size was $6.5 \mathrm{~cm}$ (range, 0 to $20.0 \mathrm{~cm}$ ), and 376 patients $(49.2 \%)$ had PVT. Before RT, the median level of AFP was $95.3 \mathrm{IU} / \mathrm{mL}$ (range, 0.3 to 2,922,754.0 $\mathrm{IU} / \mathrm{mL}$ ), and the level of protein induced by vitamin $\mathrm{K}$ absence-II was $283.5 \mathrm{mAU} / \mathrm{mL}$ (range, 7.0 to $176,595.0$ $\mathrm{mAU} / \mathrm{mL}$ ). Patients with treatment history before RT numbered $453(59.2 \%)$, and transcatheter arterial chemoembolization (TACE) or transcatheter arterial chemoinfusion (TACI) was the most common treatment before RT (51.9\%).

The treatment characteristics of patients are shown in Table 2. Tumors were included in the treatment volume in $84.4 \%$ of patients. Other patients were treated for PVT or metastatic LN. RT was performed using 3D CRT (73.9\%), intensity modulated radiotherapy (IMRT, $18.0 \%$ ), or stereotactic body radiotherapy (SBRT, 8.1\%). The median fractional dose was $2.5 \mathrm{~Gy}$ (range, 1.8 to $20.0 \mathrm{~Gy}$ ), and the median BED with $10 \mathrm{~Gy}$ of $\alpha / \beta$ was $56.3 \mathrm{~Gy}$ (range, 3.9 to $180.0 \mathrm{~Gy}$ ). In total, 733 patients $(95.8 \%)$ completed the planned RT, and the median BED (Gy 10 ) in these patients was 58.5 Gy (range, 19.5 to $180.0 \mathrm{~Gy})$. Combination with other treatment such as TACE or chemotherapy was administered in 466 patients $(60.9 \%)$

The median OS for all patients was 16.2 months, and the 2-year survival rate was $37.7 \%$ (Fig. 1). In univariate analysis, ECOG, C-P class, tumor size, number of tumors, LN metas-

Table 2. Treatment characteristics

\begin{tabular}{|cc}
\hline Characteristic & No. (\%) \\
(n=765)
\end{tabular}

PVT, portal vein thrombosis; LN, lymph node; RT, radiotherapy; 3D CRT, 3-dimensional conformal radiotherapy; IMRT, intensity-modulated radiotherapy; SBRT, stereotactic body radiotherapy; BED, biological effective dose. 


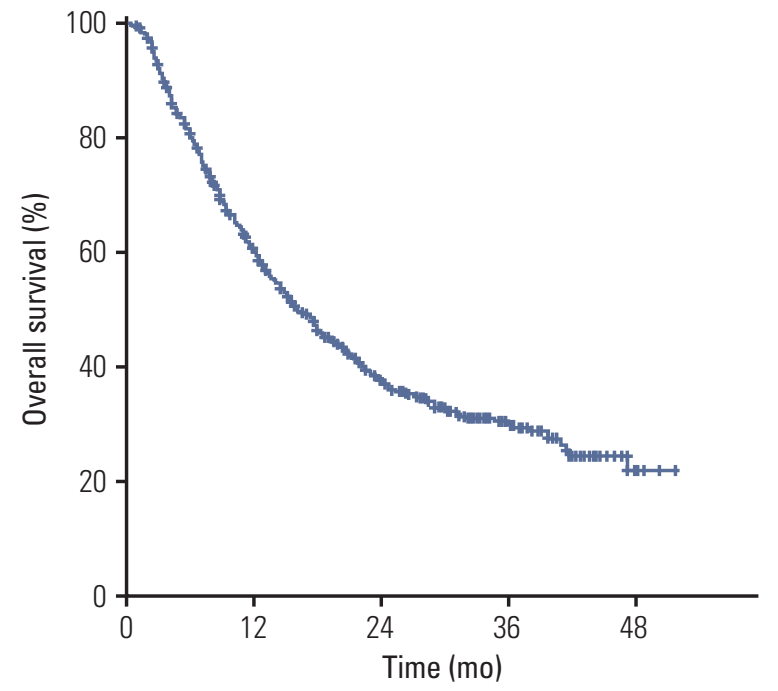

Fig. 1. Overall survival. During the follow-up period, 455 patients died. Median overall survival for all patients was 16.2 months and the 2-year survival rate was $37.7 \%$.

tasis, PVT, stage, pre-RT AFP, pre-RT treatment history, $\mathrm{BED}$, and RT technique were significant. ECOG, C-P class, tumor size, number of tumors, LN metastasis, PVT, stage, and pre-RT treatment history were significant factors in multivariate analysis (Table 3 ).

Assessment of treatment outcome was available in 734 patients (95.9\%). Infield and outfield failure occurred in 235 $(32.0 \%)$ and $547(74.5 \%)$ patients, respectively, and the 2-year FFS rate was $58.6 \%$ and $19.9 \%$ (Fig. 2). Univariate and multivariate analyses were performed for identification of predictive factors of FFS. For infield FFS, C-P class, LN metastasis, PVT, stage, BED, and RT technique were significant factors in univariate analysis, and C-P class, stage, and BED were still significant in multivariate analysis. Age, ECOG, C-P class, tumor size, and PVT were significant factors of outfield FFS, and BED showed borderline significance $(\mathrm{p}=0.053)$.

In subgroup analysis according to stage (Table 4), RT techniques were significantly different according to stage $(\mathrm{p}<$ 0.001). SBRT was used mainly in patients with early stage HCC, and most patients with advanced stage HCC were treated with 3D CRT or IMRT. Therefore, median fractional dose and BED were different between stages and were higher in early stage than advanced stage $(p<0.001)$. Combination treatment with TACE or chemotherapy was frequently administered in patients with advanced stage HCC. OS rate at 2 years in each stage was $76.7 \%, 60.4 \%, 42.9 \%$, and $22.0 \%$, respectively (Fig. 3), and survival was significantly different by stage $(\mathrm{p}<0.001)$. The 2-year infield FFS rate was $84.1 \%, 71.8 \%, 63.8 \%$, and $42.9 \%$, respectively, and the outfield
FFS rate was $12.0 \%, 13.5 \%, 6.1 \%$, and $4.9 \%$.

Compared with the previous report, patient characteristics including age, the ratio of sex, and the distribution of stage were similar. Although RT was still considered in patients with advanced HCC, the percentage of patients with good performance status increased (ECOG 0-1, 58.8\% vs. 93.3\%), and the use of RT as a first primary treatment (no pre-RT treatment) has increased (9.0\% vs. $40.8 \%)$. In terms of RT technique, two-dimensional RT was not used in this study, and use of IMRT increased (1.3\% vs. 18.0\%). Increase in IMRT resulted in an increase in radiation dose (fractional dose, 1.8 Gy vs. 2.5 Gy; BED, 53.1 Gy10 vs. 56.3 Gy10). OS was improved in all patients (median, 12 months vs. 16.2 months; 2-year OS, $27.9 \%$ vs. $37.7 \%$ ) and OS compared by stages also showed improvement (I-II, $45.2 \%$ vs. $63.4 \%$; III-IV, $24.0 \%$ vs. $33.9 \%)$.

\section{Discussion}

In this study, we demonstrated that the pattern of radiotherapy in treatment of HCC changed after revision of the guideline in 2009. Similar to the previous guideline, RT is still considered for advanced HCC. However, RT was more often used as the first primary treatment option in patients with good performance status. Use of IMRT has increased, resulting in increases in fractional dose and BED. The current patients showed better OS than those in the previous report.

Although the indication of RT was not significantly changed in the 2009 guideline, the level of evidence of RT in HCC increased from "no evidence or level 3" to "level 2." This increased level of evidence was supported by many Korean reports regarding treatment outcome of RT for patients with HCC. Loco-regional treatment was considered important and advancements in the RT technique and combination with other treatment could result in the improvement of treatment outcome, particularly in advanced HCC. Based on many evidences and experiences, referring physicians such as hepatologist or medical oncologist have gradually recognized the role of RT as the first primary treatment in patients with HCC with some learning curve period, which appeared to affect the change in pattern of RT. RT in treatment of HCC has changed from a salvage aim to a definitive aim, which might be associated with improved survival.

In the Barcelona Clinic Liver Cancer guidelines, RT is not recommended as a treatment option in patients with HCC [5]. However, the application of RT in treatment of HCC has increased, and the benefits of RT have been reported in many studies. Some authors have suggested that the application of 
Table 3. Univariate and multivariate analysis

\begin{tabular}{|c|c|c|c|c|c|c|}
\hline \multirow{2}{*}{ Variable } & \multicolumn{3}{|c|}{ Univariate } & \multicolumn{3}{|c|}{ Multivariate } \\
\hline & $\operatorname{Exp}(B)$ & $95 \% \mathrm{CI}$ & p-value & $\operatorname{Exp}(B)$ & $95 \% \mathrm{CI}$ & p-value \\
\hline \multicolumn{7}{|l|}{ Overall survival } \\
\hline Age & 0.994 & 0.986-1.003 & 0.181 & - & - & - \\
\hline Sex & 0.924 & $0.726-1.175$ & 0.517 & - & - & - \\
\hline ECOG & 3.679 & $2.707-5.001$ & $<0.001$ & 3.064 & $2.208-4.251$ & $<0.001$ \\
\hline C-P class & 3.371 & $2.752-4.129$ & $<0.001$ & 2.651 & $2.120-3.316$ & $<0.001$ \\
\hline Tumor size & 1.089 & 1.067-1.111 & $<0.001$ & 1.057 & $1.032-1.083$ & $<0.001$ \\
\hline No. of tumors & 1.466 & 1.261-1.705 & $<0.001$ & 1.264 & $1.051-1.521$ & $<0.001$ \\
\hline LN metastasis & 2.255 & $1.779-2.858$ & $<0.001$ & 1.746 & $1.332-2.291$ & $<0.001$ \\
\hline PVT & 1.931 & $1.613-2.310$ & $<0.001$ & 1.337 & $1.077-1.658$ & $<0.001$ \\
\hline Stage & 1.822 & $1.613-2.058$ & $<0.001$ & 1.320 & $1.114-1.564$ & 0.001 \\
\hline Pre-RT AFP & 1.000 & $1.000-1.000$ & $<0.001$ & - & - & - \\
\hline Pre-RT treatment & 0.825 & $0.690-0.990$ & $<0.001$ & 1.390 & $1.132-1.707$ & 0.002 \\
\hline BED & 0.984 & $0.979-0.990$ & $<0.001$ & - & - & - \\
\hline RT technique & 0.456 & $0.314-0.660$ & $<0.001$ & - & - & - \\
\hline Combination treatment & 1.105 & $0.922-1.324$ & 0.280 & - & - & - \\
\hline \multicolumn{7}{|l|}{ Infield failure-free survival } \\
\hline Age & 0.998 & $0.986-1.010$ & 0.697 & - & - & - \\
\hline Sex & 0.927 & $0.655-1.313$ & 0.670 & - & - & - \\
\hline ECOG & 1.813 & 1.034-3.179 & 0.038 & - & - & - \\
\hline C-P class & 1.740 & $1.237-2.447$ & 0.001 & 1.590 & $1.115-2.267$ & 0.010 \\
\hline Tumor size & 1.027 & 0.996-1.060 & 0.088 & - & - & - \\
\hline No. of tumors & 1.057 & $0.850-1.314$ & 0.620 & 0.706 & $0.545-0.914$ & 0.008 \\
\hline LN metastasis & 1.804 & $1.262-2.579$ & 0.001 & - & - & - \\
\hline PVT & 1.894 & $1.462-2.454$ & $<0.001$ & 1.295 & $0.972-1.726$ & 0.077 \\
\hline Stage & 1.748 & $1.468-2.082$ & $<0.001$ & 1.652 & $1.326-2.060$ & $<0.001$ \\
\hline Pre-RT AFP & 1.000 & $1.000-1.000$ & 0.639 & - & - & - \\
\hline Pre-RT treatment & 1.078 & 0.827-1.405 & 0.577 & 1.670 & $1.258-2.216$ & $<0.001$ \\
\hline BED & 0.977 & 0.969-0.986 & $<0.001$ & 0.986 & 0.977-0.995 & 0.003 \\
\hline RT technique & 0.416 & $0.237-0.729$ & 0.002 & - & - & - \\
\hline Combination treatment & 1.134 & $0.870-1.477$ & 0.352 & - & - & - \\
\hline \multicolumn{7}{|c|}{ Outfield failure-free survival } \\
\hline Age & 0.988 & 0.980-0.996 & 0.002 & 0.991 & 0.983-0.999 & 0.036 \\
\hline Sex & 0.920 & $0.735-1.153$ & 0.470 & - & - & - \\
\hline ECOG & 2.167 & $1.512-3.106$ & $<0.001$ & 1.791 & $1.222-2.625$ & 0.003 \\
\hline C-P class & 1.735 & $1.388-2.168$ & $<0.001$ & 1.510 & $1.184-1.925$ & 0.001 \\
\hline Tumor size & 1.046 & $1.025-1.067$ & $<0.001$ & 1.035 & $1.010-1.061$ & 0.007 \\
\hline No. of tumors & 1.349 & $1.171-1.555$ & $<0.001$ & 1.185 & 0.996-1.409 & 0.055 \\
\hline LN metastasis & 1.418 & $1.104-1.821$ & $<0.001$ & - & - & - \\
\hline PVT & 1.470 & $1.241-1.740$ & $<0.001$ & 1.229 & $1.002-1.507$ & 0.048 \\
\hline Stage & 1.359 & $1.223-1.509$ & $<0.001$ & 1.142 & $0.985-1.325$ & 0.078 \\
\hline Pre-RT AFP & 1.000 & $1.000-1.000$ & $<0.001$ & - & - & - \\
\hline Pre-RT treatment & 1.086 & 0.914-1.290 & 0.348 & 1.349 & $1.075-1.692$ & 0.010 \\
\hline BED & 0.990 & 0.986-0.995 & $<0.001$ & 0.995 & $0.989-1.000$ & 0.053 \\
\hline RT technique & 0.653 & $0.478-0.892$ & 0.007 & - & - & - \\
\hline Combination treatment & 0.923 & 0.778-1.095 & 0.360 & 0.796 & $0.637-0.993$ & 0.044 \\
\hline
\end{tabular}

CI, confidence interval; ECOG, Eastern Cooperative Oncology Group; C-P, Child-Pugh; LN, lymph node; PVT, portal vein thrombosis; RT, radiotherapy; AFP, $\alpha$-fetoprotein; BED, biological effective dose. 


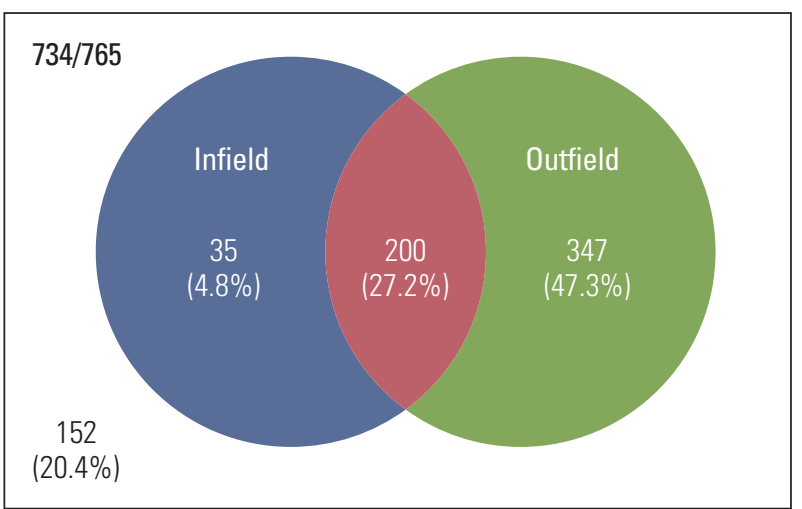

B
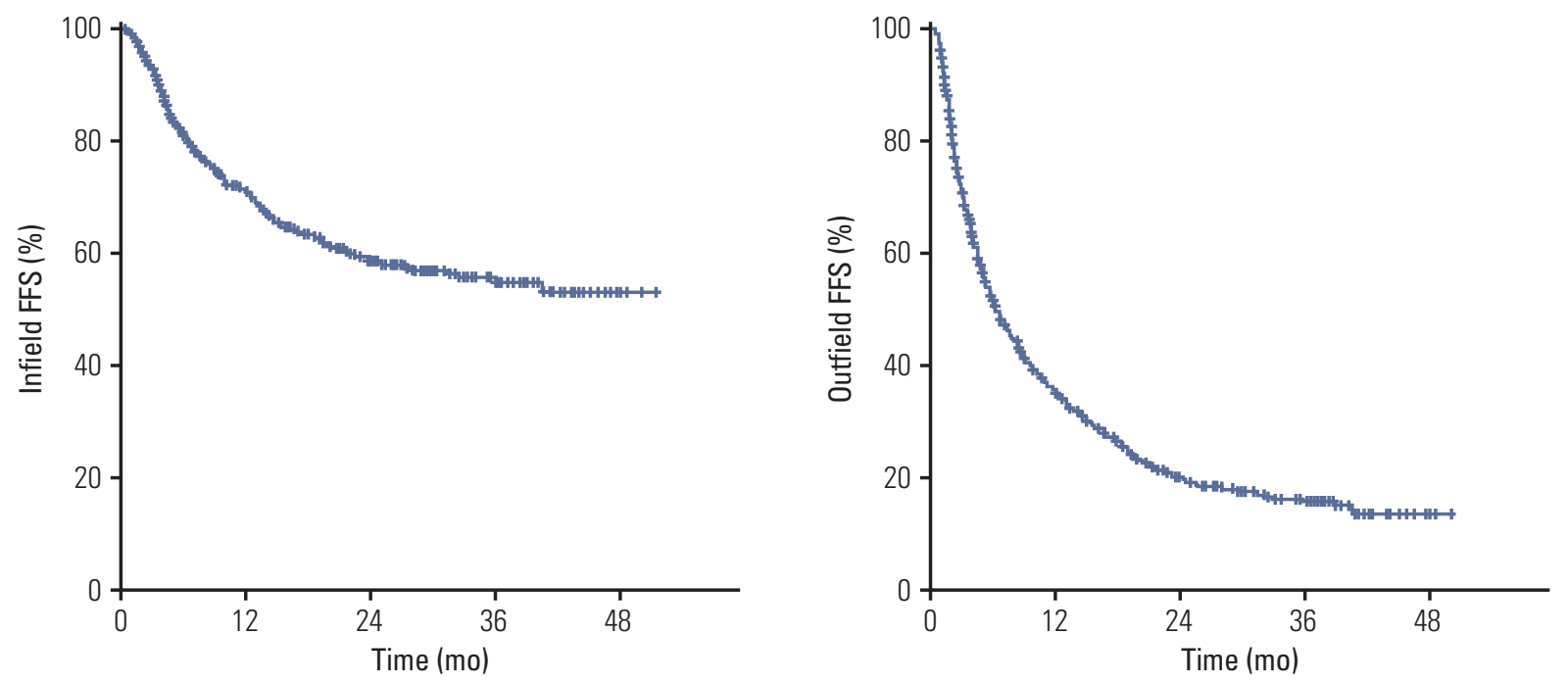

Fig. 2. (A) Pattern of failure. Assessment of treatment outcome was available in 734 patients (95.9\%). Infield and outfield failure occurred in $235(32.0 \%)$ and $547(74.5 \%)$ patients. (B) Failure-free survival (FFS) for infield failure. Median FFS was not reached and 2-year FFS rate was 58.6\%. (C) FFS for outfield failure. Median FFS was 9.8 months and 2-year FFS was $19.9 \%$.

RT according to the BCLC staging system could be used in all stages of HCC [6-8]. In very early or early stage disease, SBRT alone could be considered, and combination with TACE is also possible if the patient is inoperable or not eligible for curative treatment such as surgery or radiofrequency ablation (RFA). Combination with other treatment was recommended in intermediate or advanced stage. Palliative RT for symptom relief was recommended for terminal patients.

In 2014, the KLCSG-NCC revised the practice guidelines for the management of HCC [9]. In addition to the indication of the previous guideline, the revised guideline suggests that external beam radiotherapy can be considered as an alternative treatment option in early stage disease. In patients with early HCC who are ineligible for surgical resection, liver transplantation, RFA, percutaneous ethanol injection, or TACE and the tumor is far from a radiosensitive normal organ, SBRT could be an effective treatment.

The outcome of SBRT for HCC was reported in previous studies $[10,11]$. SBRT showed high local control for inoperable HCC patients and resulted in a survival benefit. Accord- 
Table 4. Treatment characteristics according to stage

\begin{tabular}{|c|c|c|c|c|c|}
\hline Characteristic & $\begin{array}{c}\text { Stage I } \\
(n=29)\end{array}$ & $\begin{array}{l}\text { Stage II } \\
(n=111)\end{array}$ & $\begin{array}{c}\text { Stage III } \\
(n=287)\end{array}$ & $\begin{array}{c}\text { Stage IV } \\
(n=338)\end{array}$ & p-value \\
\hline \multicolumn{6}{|l|}{ RT technique } \\
\hline 3D CRT & $13(44.8)$ & $61(55.0)$ & $228(79.4)$ & $263(77.8)$ & $<0.001$ \\
\hline IMRT & $1(3.4)$ & $24(21.6)$ & $43(15.0)$ & $70(20.7)$ & \\
\hline SBRT & $15(51.7)$ & $26(23.4)$ & $16(5.6)$ & $5(1.5)$ & \\
\hline Fractional dose (Gy) & $7.0(2.0-19.0)$ & $3.5(1.8-20.0)$ & $2.5(1.8-20.0)$ & $2.5(1.8-13.0)$ & $<0.001$ \\
\hline $\operatorname{BED}\left(\mathrm{Gy}_{10}\right)$ & $79.2(42.9-165.3)$ & $65.3(37.5-180.0)$ & $56.0(3.9-180.0)$ & $53.1(14.4-119.6)$ & $<0.001$ \\
\hline \multicolumn{6}{|c|}{ Completion of planned RT } \\
\hline No & 0 & $1(0.9)$ & $7(2.4)$ & $24(7.1)$ & 0.005 \\
\hline Yes & $29(100)$ & $110(99.1)$ & $280(97.6)$ & $314(92.9)$ & \\
\hline \multicolumn{6}{|c|}{ Combination treatment } \\
\hline No & $26(89.7)$ & $71(64.0)$ & $98(34.1)$ & $104(30.8)$ & $<0.001$ \\
\hline Yes & $3(10.3)$ & $40(36.0)$ & $189(65.9)$ & $234(69.2)$ & \\
\hline
\end{tabular}

Values are presented as number (\%) or median (range). RT, radiotherapy; 3D CRT, 3-dimensional conformal radiotherapy; IMRT, intensity-modulated radiotherapy; SBRT, stereotactic body radiotherapy; BED, biological effective dose.

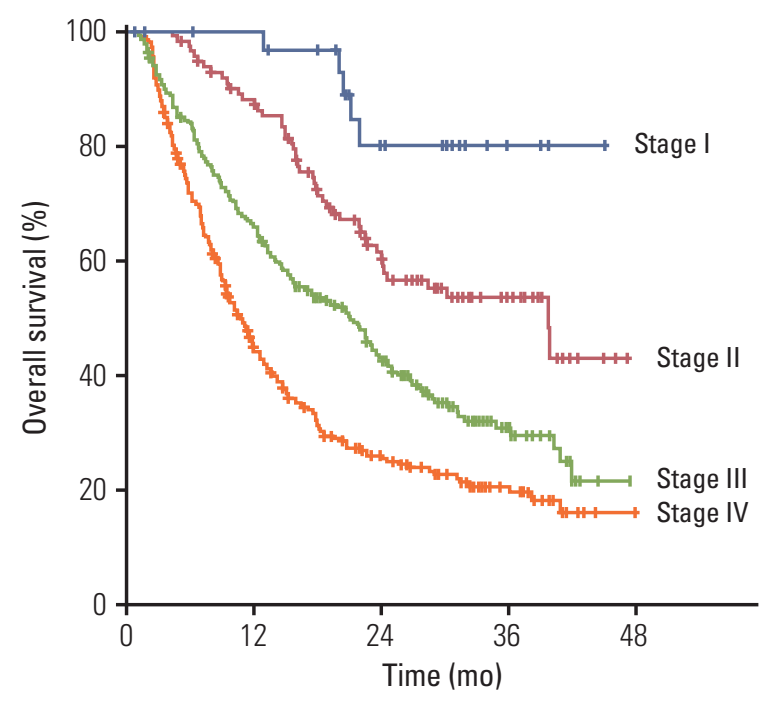

Fig. 3. Overall survival according to stage. Overall survival rates at 2 years in each stage were $76.7 \%, 60.4 \%$, $42.9 \%$, and $22.0 \%$, respectively, and it was significantly different by stage $(\mathrm{p}<0.001)$.

ing to a nationwide survey conducted in Korea, the application of SBRT has increased, and the liver is a common treatment site [12]. In this study, the percentage of patients treated with SBRT was similar to that in a previous report, and SBRT was used mainly as a salvage treatment for recurrent tumors with early stage. Unfortunately, detailed data from previous study could not be obtained for specific comparison of RT dose. SBRT was introduced in the previous era of study and has now become popular throughout the nation. It can be postulated that dose of SBRT was not sufficient in the previous study period, and the experience and advancement of the SBRT technique became available for more delicate treatment, which could contribute to survival gain in early stage.

Combination treatment was administered in 466 patients $(60.9 \%)$ who were considered mainly in advanced stage rather than early stage HCC. TACE or TACI was administered in $41.9 \%$ of patients, and hepatic arterial infusional chemotherapy (HAIC) was administered in $23.1 \%$ of patients. Radiotherapy following TACE is known to be effective [13-15]. A prospective phase 2 multicenter study conducted in Korea demonstrated that TACE plus RT is a safe and effective treatment in patients with unresectable HCC. Shim et al. [15] reported that TACE+RT showed better survival than TACE alone, particularly in patients with tumors $\geq 8 \mathrm{~cm}$ in diameter. Concurrent chemoradiotherapy (CCRT) using HAIC is a treatment option in locally advanced HCC $[16,17]$. Overall tumor response after CCRT was approximately $60 \%$, and response in both tumor and portal vein tumor thrombosis showed significant correlation with improved survival.

Due to improvement in RT techniques, two-dimensional conventional RT is no longer used, and the use of IMRT has increased. Although the median total dose was not different from that in the previous report, fractional dose and BED increased in the current study, possibly due to an increase in IMRT. IMRT for HCC has shown a good response without severe complications in several studies [18-20]. Yoon et al. [19] compared treatment outcomes between patients with locally advanced HCC treated with 3D CRT and those treated with IMRT by propensity score matching method. Patients treated with IMRT showed better survival than 
those treated with 3D CRT, and RT modality was a significant factor of OS [19]. Kim et al. [20], who reported the results of simultaneous integrated boost-IMRT (SIB-IMRT) in patients with inoperable HCC, suggested that SIB-IMRT was a feasible and safe treatment for patients with inoperable HCC. However, there was no significant difference in survival or treatment failure between 3D CRT and IMRT in the current study. Based on a retrospective multi-institutional review, it was thought to be due to the heterogeneity of patient and treatment characteristics. In 2015, the indications of IMRT were expanded to treatment for patients with HCC, and it is thought that the use of IMRT will further increase.

Tumor size, LN metastasis, C-P class, and BED were significant factors of OS in the previous report. Similarly, ECOG, C-P class, tumor size, number of tumors, PVT, and stage were significant factors of OS in the current study. Tumor factors and host factors (performance status, liver function) are important for survival outcome. BED was not a significant factor of OS; however it was a significant factor of infield failure.

Although this study was not a complete enumeration survey and conducted in 12 institutions, most institutions that treat a large number of HCC patients with RT were included and nine of 12 institutions are included in both the previous and current study. This study was based on a retrospective review in multiple institutions; therefore, complete data regarding toxicity or quality of life could not be obtained. However, evaluation of treatment outcome was available in most patients (95.9\%), while the previous study reported disease status in only one-third of all patients. The result of this study does not represent the practice pattern of all HCC patients with regard to RT in Korea; however, it shows increased understanding among members of the hepatic oncologists society in terms of RT application, which expands to the primary aim.

Further study examining changes in patterns of RT in HCC is required after revision of the KLCSG-NCC guideline in 2014.

\section{Conclusion}

By increasing the level of evidence of RT in the KLCSGNCC guideline, clinical practice patterns of RT have changed. RT is still used mainly in advanced HCC; however, the number of patients with good performance status who received $\mathrm{RT}$ as a first treatment has increased. This change in practice patterns could result in improvement in OS.

\section{Conflicts of Interest}

Conflict of interest relevant to this article was not reported.

\section{Acknowledgments}

This study was supported by a grant from the Korean Health Technology R\&D Project (A121982), Ministry of Health \& Welfare, Republic of Korea.

\section{Author Details}

${ }^{1}$ Department of Radiation Oncology, Yonsei Cancer Center, Yonsei University College of Medicine, Seoul, ${ }^{2}$ Department of Radiation Oncology, Samsung Medical Center, Sungkyunkwan University School of Medicine, Seoul, ${ }^{3}$ Department of Medical Device Management and Research, SAIHST, Sungkyunkwan University, Seoul, ${ }^{4}$ Research Institute and Hospital, National Cancer Center, Goyang, ${ }^{5}$ Department of Radiation Oncology, Chonnam National University Medical School, Gwangju, ${ }^{6}$ Department of Radiation Oncology, Asan Liver Center, Asan Medical Center, University of Ulsan College of Medicine, Seoul, ${ }^{7}$ Department of Radiation Oncology, Korea University Ansan Hospital, Ansan, ${ }^{8}$ Department of Radiation Oncology, Gangnam Severance Hospital, Yonsei University College of Medicine, Seoul, ${ }^{9}$ Department of Radiation Oncology, Korea Institute of Radiological and Medical Sciences, Seoul, ${ }^{10}$ Department of Radiation Oncology, College of Medicine, The Catholic University of Korea, Seoul, ${ }^{11}$ Department of Radiation Oncology, Dong-A University College of Medicine, Busan, ${ }^{12}$ Department of Radiation Oncology, Keimyung University Dongsan Medical Center, Keimyung University School of Medicine, Daegu, ${ }^{13}$ Department of Radiation Oncology, Incheon St. Mary's Hospital, College of Medicine, The Catholic University of Korea, Incheon, ${ }^{14}$ Department of Biostatistics, Severance Hospital, Yonsei University College of Medicine, Seoul, Korea 


\section{References}

1. Jung KW, Won YJ, Kong HJ, Oh CM, Cho H, Lee DH, et al. Cancer statistics in Korea: incidence, mortality, survival, and prevalence in 2012. Cancer Res Treat. 2015;47:127-41.

2. Park JW; Korean Liver Cancer Study Group and National Cancer Center. Practice guideline for diagnosis and treatment of hepatocellular carcinoma. Korean J Hepatol. 2004;10:88-98.

3. Seong J, Lee IJ, Shim SJ, Lim DH, Kim TH, Kim JH, et al. A multicenter retrospective cohort study of practice patterns and clinical outcome on radiotherapy for hepatocellular carcinoma in Korea. Liver Int. 2009;29:147-52.

4. Korean Liver Cancer Study Group and National Cancer Center. Practice guidelines for management of hepatocellular carcinoma 2009. Korean J Hepatol. 2009;15:391-423.

5. Forner A, Reig ME, de Lope CR, Bruix J. Current strategy for staging and treatment: the BCLC update and future prospects. Semin Liver Dis. 2010;30:61-74.

6. Dawson LA. Overview: where does radiation therapy fit in the spectrum of liver cancer local-regional therapies? Semin Radiat Oncol. 2011;21:241-6.

7. Lee IJ, Seong J. Radiotherapeutic strategies in the management of hepatocellular carcinoma. Oncology. 2011;81 Suppl 1:12333.

8. Cha J, Seong J. Application of radiotherapeutic strategies in the BCLC-defined stages of hepatocellular carcinoma. Liver Cancer. 2012;1:216-25.

9. Korean Liver Cancer Study Group (KLCSG); National Cancer Center, Korea (NCC). 2014 KLCSG-NCC Korea practice guideline for the management of hepatocellular carcinoma. Gut Liver. 2015;9:267-317.

10. Jang WI, Kim MS, Bae SH, Cho CK, Yoo HJ, Seo YS, et al. High-dose stereotactic body radiotherapy correlates increased local control and overall survival in patients with inoperable hepatocellular carcinoma. Radiat Oncol. 2013;8:250.

11. Huertas A, Baumann AS, Saunier-Kubs F, Salleron J, Oldrini G, Croise-Laurent V, et al. Stereotactic body radiation therapy as an ablative treatment for inoperable hepatocellular carcinoma. Radiother Oncol. 2015;115:211-6.

12. Bae SH, Kim MS, Jang WI, Kay CS, Kim W, Kim ES, et al. A survey of stereotactic body radiotherapy in Korea. Cancer Res Treat. 2015;47:379-86.

13. Choi C, Koom WS, Kim TH, Yoon SM, Kim JH, Lee HS, et al. A prospective phase 2 multicenter study for the efficacy of radiation therapy following incomplete transarterial chemoembolization in unresectable hepatocellular carcinoma. Int J Radiat Oncol Biol Phys. 2014;90:1051-60.

14. Cho JY, Paik YH, Park HC, Yu JI, Sohn W, Gwak GY, et al. The feasibility of combined transcatheter arterial chemoembolization and radiotherapy for advanced hepatocellular carcinoma. Liver Int. 2014;34:795-801.

15. Shim SJ, Seong J, Han KH, Chon CY, Suh CO, Lee JT. Local radiotherapy as a complement to incomplete transcatheter arterial chemoembolization in locally advanced hepatocellular carcinoma. Liver Int. 2005;25:1189-96.

16. Cha H, Yoon HI, Lee IJ, Koom WS, Han KH, Seong J. Clinical factors related to recurrence after hepatic arterial concurrent chemoradiotherapy for advanced but liver-confined hepatocellular carcinoma. J Radiat Res. 2013;54:1069-77.

17. Choi Y, Kim JW, Cha H, Han KH, Seong J. Overall response of both intrahepatic tumor and portal vein tumor thrombosis is a good prognostic factor for hepatocellular carcinoma patients receiving concurrent chemoradiotherapy. J Radiat Res. 2014;55:113-20.

18. Kang MK, Kim MS, Kim SK, Ye GW, Lee HJ, Kim TN, et al. High-dose radiotherapy with intensity-modulated radiation therapy for advanced hepatocellular carcinoma. Tumori. 2011; 97:724-31.

19. Yoon HI, Lee IJ, Han KH, Seong J. Improved oncologic outcomes with image-guided intensity-modulated radiation therapy using helical tomotherapy in locally advanced hepatocellular carcinoma. J Cancer Res Clin Oncol. 2014;140:1595605.

20. Kim TH, Park JW, Kim YJ, Kim BH, Woo SM, Moon SH, et al. Simultaneous integrated boost-intensity modulated radiation therapy for inoperable hepatocellular carcinoma. Strahlenther Onkol. 2014;190:882-90. 